\title{
UNIVERSAL VECTORS FOR OPERATORS ON SPACES OF HOLOMORPHIC FUNCTIONS
}

\author{
ROBERT M. GETHNER AND JOEL H. SHAPIRO
}

\begin{abstract}
A vector $x$ in a linear topological space $X$ is called universal for a linear operator $T$ on $X$ if the orbit $\left\{T^{n} x: n \geq 0\right\}$ is dense in $X$. Our main result gives conditions on $T$ and $X$ which guarantee that $T$ will have universal vectors. It applies to the operators of differentiation and translation on the space of entire functions, where it makes contact with Pólya's theory of final sets; and also to backward shifts and related operators on various Hilbert and Banach spaces.
\end{abstract}

1. Introduction. Suppose $T$ is a continuous linear operator on a complete metrizable linear topological space (henceforth an $F$-space) $X$. We say that $x \in X$ is $T$-universal if its orbit $\left\{T^{n} x: n \geq 0\right\}$ is dense in $X$, and $T$-cyclic if the linear span of its orbit is dense. Cyclic vectors play an important role in the study of the invariant subspace structure of an operator, and universal vectors are cyclic in the strongest possible sense.

The main result of this paper, Theorem 2.2, gives hypotheses which guarantee that $T$ will have universal vectors. Its proof, which is based on the Baire Category Theorem, actually provides a dense $G_{\delta}$ set of universal vectors. However this is no surprise: it is easy to see (Proposition 2.1) that if $T$ has any universal vectors, then it has a dense $G_{\delta}$ set of them.

Interest in Theorem 2.2 derives from the fact that it unifies, extends, and complements diverse results which have occurred in the literature of both classical function theory and operator theory.

For example $H(\mathbf{C})$, the space of entire functions taken in the compact-open topology, is a separable $F$-space ([4, Chapter VII $]$ ) on which both the operator of translation by a fixed complex number and that of complex differentiation satisfy the hypotheses of Theorem 2.2. Thus we obtain at one stroke proofs of G. D. Birkhoff's translation theorem $[1,2,3]$ : There exists an entire function whose translates are dense in $H(\mathbf{C})$, and G. R. MacLane's differentiation theorem [10, Theorem 7]: There exist entire functions $f$ for which the sequence of successive derivatives is dense in $H(\mathbf{C})$. We get equally quick proofs of some generalizations due to Luh [8], and Seidel and Walsh [15] of Birkhoff's theorem. We use our results to complement existing work of Pólya [11] and Edrei and MacLane [6], by showing that the "final set" $L(f)$ of accumulation points of zeros of the sequence of derivatives of an entire function is generically (meaning "except for a set of first

Received by the editors August 2, 1985 and, in revised form, March 31, 1986.

1980 Mathematics Subject Classification (1985 Revision). Primary 30D20, 47B73; Secondary 30D35, $30 \mathrm{C} 15$.

Key words and phrases. Cyclic vector, entire function, backward shift, final set, successive derivatives. 
Baire category") the whole Riemann sphere. A detailed discussion of these matters occupies the third section.

$\S 4$ contains applications to operator theory. Each backward shift of "Bergman type" on Hilbert space satisfies the hypotheses of Theorem 2.2, and thus has universal vectors, as does every multiple, by a scalar of modulus $>1$, of the ordinary backward shift. Our work also intersects that of Rolewicz [13], who showed that on the sequence spaces $l^{p}(1 \leq p<\infty)$ and $c_{0}$ certain multiples of the backward shift have universal vectors.

We wish to take this opportunity to thank Sheldon Axler, Paul Bourdon, Lech Drewnowski, Lee Rubel, and Allen Shields for helpful discussions and useful references to the literature.

2. Universal vectors. In all that follows, $X$ is a separable $F$-space and $T$ a continuous linear operator on $X$. The topology of $X$ is induced by a complete translation-invariant metric $d$ [14, Theorems 1.24 and 1.26, pp. 18-20]. For $x \in X$ we write $\|x\|=d(x, 0)$, and let $B(y, \varepsilon)=\{x \in X:\|y-x\|<\varepsilon\}$, the open ball of radius $\varepsilon$ with center at $y$. Before stating our main theorem, we record a simple, but useful, "zero-one" law for universal vectors (cf. Duios-Ruis [18]).

2.1. Proposition. If $T$ has a universal vector, then it has a dense $G_{\delta}$ set of universal vectors.

ProOF. Fix a countable dense subset $\left\{y_{k}\right\}$ of $X$. For positive integers $N, j$, and $k$, set

$$
F=F(j, N, k)=\left\{x \in X:\left\|T^{n} x-y_{j}\right\|<1 / k \text { for some } n \geq N\right\} .
$$

Each of these sets, being a union of sets $T^{-n}\left\{B\left(y_{j}, 1 / k\right)\right\}$ which are open by the continuity of $T$, is itself open. The set of $T$-universal vectors is the intersection of this countable collection of sets $F$ : it is therefore a $G_{\delta}$ subset of $X$. If $x$ is a universal vector, then so is every member of the dense orbit $\left\{T^{n} x\right\}$. This completes the proof.

A curious consequence of this result and Baire's Theorem is that if each member of a countable collection of operators has a universal vector, then the whole collection has a common universal vector. Thus, for example, the previously mentioned theorems of Birkhoff and MacLane, providing respectively translation- and differentiation-universal entire functions, automatically provide a "doubly universal" entire function (see also [3] for the direct construction of a "triply universal" entire function).

We now turn to our main result.

2.2. THEOREM. Suppose $T$ is a continuous linear operator on a separable $F$-space $X$. Suppose there exists a dense subset $D$ of $X$ and a right inverse $S$ for $T(T S=$ identity on $X)$ such that $\left\|T^{n} x\right\| \rightarrow 0$ and $\left\|S^{n} x\right\| \rightarrow 0$ for every $x \in D$. Then $X$ has $T$-universal vectors.

Proof. By Baire's Theorem $[\mathbf{1 4}$, p. 42] it is enough to prove that each of the $G_{\delta}$ sets $F$ defined in the proof of Proposition 2.1 is dense in $X$.

To see this, fix $F=F(j, N, k)$, and for ease of notation write $\varepsilon=1 / k$ and $y=y_{j}$. Fix $z$ in $X$ and $\delta>0$. We must find an $x \in F$ lying within $\delta$ of $z$. Since $D$ is dense in $X$, we can choose $y_{0}$ and $z_{0}$ in $D$ with $\left\|z-z_{0}\right\|<\delta / 2$, and $\left\|y-y_{0}\right\|<\varepsilon / 2$. 
Since the sequences $T^{n}$ and $S^{n}$ converge pointwise to zero on $D$, we may choose a positive integer $n$ such that simultaneously $\left\|T^{n} z_{0}\right\|<\varepsilon / 2$, and $\left\|S^{n} y_{0}\right\|<\delta / 2$. Write $x=S^{n} y_{0}+z_{0}$. Then

$$
\|x-z\| \leq\left\|x-z_{0}\right\|+\left\|z_{0}-z\right\|=\left\|S^{n} y_{0}\right\|+\left\|z_{0}-z\right\|<\delta / 2+\delta / 2,
$$

so $\|x-z\|<\delta$, as desired. Moreover, since $T S$ is the identity map on $X$, so is $T^{n} S^{n}$. Thus

$$
\left\|T^{n} x-y\right\|=\left\|T^{n} S^{n} y_{0}-y+T^{n} z_{0}\right\| \leq\left\|y_{0}-y\right\|+\left\|T^{n} z_{0}\right\|<\varepsilon / 2+\varepsilon / 2=\varepsilon,
$$

so $x \in F$, and the proof is complete.

2.3. REMARKS. (a) The proof actually shows that for any fixed subsequence $\{n(j): j \geq 0\}$ of positive integers increasing strictly to $\infty$, the sequence of vectors $\left\{T^{n(j)} x: j \geq 0\right\}$ is dense in $X$ for a dense $G_{\delta}$ subset of $x$ 's. In fact Lech Drewnowski has pointed out to us that the proof yields even more. Suppose $D$ is a dense subset of $X$ and $\left\{T_{j}\right\}$ is a sequence of continuous linear operators on $X$ for which $T_{j} \rightarrow 0$ pointwise on $D$. Suppose for each $j$ the operator $T_{j}$ has a right inverse $S_{j}$, and $S_{j} \rightarrow 0$ pointwise on $D$. Then the set $\left\{T_{j} x: j \geq 0\right\}$ is dense in $X$ for a dense $G_{\delta}$ set of vectors $x \in X$.

(b) Theorem 2.2, and the remark above, continue to hold with almost the same proof, if the sequences of $T$ 's and $S$ 's are assumed to converge to zero pointwise on different dense subsets of $X$. However, none of the applications given here require this generality.

Here is another variant of Theorem 2.2. It will also be useful in the next section: its proof is a straightforward modification of the original one.

2.4. THEOREM. Suppose $T$ is a continuous linear operator on a separable $F$-space $X$, and $T^{n} \rightarrow 0$ pointwise on a dense subset $D_{0}$ of $X$. Suppose $\left\{y_{n}\right\}$ is a sequence in $X$ such that $y_{n}=T^{n} x_{n}$ where $x_{n} \rightarrow 0$ in $X$. Then the set of vectors $x \in X$ for which liminf $\left\|T^{n} x-y_{n}\right\|=0$ is a dense $G_{\delta}$ subset of $X$.

ProOF. Let $F(N, \varepsilon)$ denote all $x$ in $X$ for which $\left\|T^{n} x-y_{n}\right\|<\varepsilon$ for some $n>N$ : the set of vectors obeying the desired conclusion is then $\bigcap_{N} \bigcap_{k} F(N, 1 / k)$, so it is enough to prove that $F=F(N, \varepsilon)$ is a dense $G_{\delta}$. To do this, begin with $z, \delta$, and $z_{0}$ as in the proof of the Main Theorem, and as before, observe that for sufficiently large $n$ the vector $x=x_{n}+z_{0}$ belongs to $B(z, \delta) \cap F$.

3. Applications to function theory. Following Blair and Rubel $[2,3]$ and Luh $[\mathbf{8}, \mathbf{9}]$ we call an entire function $f$ universal if its sequence $\left\{f^{(n)}: n \geq 0\right\}$ of successive derivatives is dense in $H(\mathbf{C})$.

3.1. MACLANE'S THEOREM $[\mathbf{2}, \mathbf{1 0}]$. The universal entire functions form a dense $G_{\delta}$ subset of $H(\mathbf{C})$.

ProOF. The hypotheses of Theorem 2.2 are satisfied with $X=H(\mathbf{C}), D$ the set of holomorphic polynomials, $T$ the operator of complex differentiation, and $S$ the integration operator defined for $z_{0}$ fixed in $\mathbf{C}$ by

$$
S f(z)=\int_{z_{0}}^{z} f(\varsigma) d \varsigma \quad(f \in H(\mathbf{C}), z \in \mathbf{C}) .
$$

If $f$ is an entire function and $\alpha \in \mathbf{C}$, let $f_{\alpha}$ denote the translate of $f$ by $\alpha$ :

$$
f_{\alpha}(z)=f(z+\alpha) \quad(z \in \mathbf{C}) .
$$


3.2. BIRKHOFF'S THEOREM [1]. For each $\alpha \in \mathbf{C}$ there is a dense $G_{\delta}$ set of entire functions $f$ such that the translates $\left\{f_{n \alpha}: n=1,2, \ldots\right\}$ are dense in $H(\mathbf{C})$.

Proof. We are going to apply Theorem 2.2 to $X=H(\mathbf{C})$ with $T$ the operator of translation by $\alpha\left(T f=f_{\alpha}\right.$ for each $\left.f \in H(\mathbf{C})\right)$, and $S$ the operator of translation by $-\alpha$. The problem is to find a dense set on which the powers of these operators tend pointwise to zero. For this, we may without loss of generality, assume that $\alpha$ is real. Then, for each pair of integers $k>0$ and $m \geq 0$, define the entire function $f_{m, k}$ by

$$
f_{m, k}(z)=z^{m}\left[(z / k)^{-1} \sin (z / k)\right]^{m+1} \quad(z \in \mathbf{C}) .
$$

For $m$ and $k$ fixed, both $T^{n} f_{m, k}$ and $S^{n} f_{m, k} \rightarrow 0$ in $H(\mathbf{C})$ as $n \rightarrow \infty$. Thus $\left\{T^{n}\right\}$ and $\left\{S^{n}\right\}$ tend pointwise to zero on the linear span $D$ of these functions. It remains to show that $D$ is dense in $H(\mathbf{C})$. This is so because, if $m \geq 0$ is fixed, then $f_{m, k}(z) \rightarrow z^{m}$ uniformly on compact subsets of $\mathbf{C}$ as $k \rightarrow \infty$. Thus all the hypotheses of Theorem 2.2 are fulfilled, and the proof is complete.

3.3. REMARKS. (a) The same argument, with Drewnowski's Remark 2.3 replacing Theorem 2.2, proves the following generalization, due to Luh [8] of Birkhoff's theorem. If $E$ is any unbounded set of real numbers, then there is a dense $G_{\delta}$ set of entire functions $f$ for which the sequence $\left\{f_{\alpha}: \alpha \in E\right\}$ is dense in $H(\mathbf{C})$. S. M. Duios-Ruis [17] has constructed universal entire functions satisfying prescribed growth conditions.

(b) There are non-Euclidean analogues of Birkhoff's theorem for the unit disc $U$, and hence for any simply connected domain. In [15], Seidel and Walsh prove the following. Suppose $\left\{a_{n}\right\}$ is a sequence of points in $U$ with $a_{n} \rightarrow 1$. For each $n$ let $\varphi_{n}$ be the conformal automorphism of $U$ defined by

$$
\varphi_{n}(z)=\left(a_{n}-z\right) /\left(1-\bar{a}_{n} z\right) \quad(z \in U) .
$$

Then there exists a function $f \in H(U)$ for which the sequence of non-Euclidean translates $\left\{f \circ \varphi_{n}: n \geq 0\right\}$ is dense in $H(U)$.

This result follows easily from Remark 2.3: noting that each conformal automorphism defined above is its own inverse, we can set $T_{n}=S_{n}=$ composition with $\varphi_{n}$. Let $D$ denote the linear span of the functions

$$
f_{m, k}(z)=z^{m}\left(1-z^{k}\right) /\left(1+z^{k}\right) \quad(z \in U, m \geq 0, k>0) .
$$

Then for fixed $m$ as $k \rightarrow \infty$ the sequence $f_{m, k}$ tends to the function $z^{m}$ uniformly on compact subsets of $U$, so $D$ is dense in $H(U)$. Since $\varphi_{n} \rightarrow 1$ uniformly on compact subsets of $U$, and each member of $D$ is holomorphic in a neighborhood of 1 and vanishes at 1 , it follows that $T_{n} \rightarrow 0$ pointwise on $D$. Thus the hypotheses of Remark 2.3 are satisfied, and the theorem of Seidel and Walsh is proved.

Luh [9] has proved other results of this type.

3.4. Final sets. Pólya $[\mathbf{1 1}, \mathbf{1 2}]$ initiated the study of the final set of a function meromorphic in the plane. This is the set $L(f)$ of points of the Riemann sphere $\mathbf{C}^{*}$, each neighborhood of which contains zeros of infinitely many derivatives of $f$. Pólya showed that if $f$ is not entire, then its final set is determined completely by its poles, and consists of a union of rays, lines, and line segments. However Edrei and MacLane [6] showed that for entire functions the situation is much more complicated: every closed subset of $\mathbf{C}^{*}$ that contains $\infty$ is the final set of some entire function. By contrast, the next result shows that generically $L(f)=\mathbf{C}^{*}$. 
3.5. Corollary. $L(f)=\mathbf{C}^{*}$ for all $f$ in a dense $G_{\delta}$ subset of $H(\mathbf{C})$.

ProOF. In fact the dense $G_{\delta}$ subset is the collection of universal entire functions. Suppose $f \in H(\mathbf{C})$ is universal. Fix $z_{0} \in \mathbf{C}$. Since $f$ is universal, some subsequence of its derivatives converges uniformly on compact subsets to the entire function $z-z_{0}$, so by Hurwitz's theorem [3, Chapter VII, p. 152], each disc centered at $z_{0}$ contains zeros of all but finitely many members of this subsequence. Thus $z_{0} \in L(f)$. This shows that $\mathbf{C} \subset L(f)$, hence also $\infty \in L(f)$. The proof is complete.

3.6. REMARK. The last proof shows a little more. For $a \in \mathbf{C}$ let $L(a, f)$ denote the collection of sequential limit points of the a-points of the derivatives of $f$, so in this notation the final set of $f$ is now $L(f, 0)$. Pólya [11] showed that if $f$ is meromorphic in the plane and not entire, then $L(a, f)=L(f)$ for all complex numbers $a$. The proof above, with the function $z-z_{0}$ replaced by $z-z_{0}+a$, yields: for every $a \in \mathbf{C}, L(a, f)=\mathbf{C}^{*}$ for every universal entire function $f$.

The work of this section shows that generically the sequence of derivatives of an entire function behaves wildly. We close with an even stronger result of this type. In what follows, $D(w, r)$ denotes the open disc in the plane of radius $r$, centered at $w$.

3.7. THEOREM. Suppose $\left\{\rho_{n}\right\}$ is an unbounded, increasing sequence of positive numbers for which

(1) $\lim n^{-1} \rho_{n}^{1 / n}=0(n \rightarrow \infty)$.

Let $F$ be the set of entire functions $f$ such that

(2) for every open set $V \subset \mathbf{C} ; f^{(n)}(V) \supset D\left(0, \rho_{n}\right)$ for infinitely many $n$.

Then $F$ is a dense $G_{\delta}$ subset of $H(\mathbf{C})$.

ProOF. We begin with a fixed disc $\Delta=D(w, \varepsilon)$, and associate to it the sequence of degree one monomials $\left\{g_{n}\right\}$ defined by

$$
g_{n}(z)=n \rho_{n}(z-w) \quad(z \in \mathbf{C}) .
$$

Let $\Gamma(\Delta)$ denote the set of entire functions $f$ for which some subsequence of $\left\{f^{(n)}\right.$ $\left.g_{n}\right\}$ tends to zero uniformly on compact subsets of the plane.

We claim that $\Gamma(\Delta)$ is a dense $G_{\delta}$ subset of $H(\mathbf{C})$. To see this, let

$$
h_{n}(z)=\left[n \rho_{n} /(n+1) !\right](z-w)^{n+1} \quad(z \in \mathbf{C}) .
$$

Then $h_{n}^{(n)}=g_{n}$ for each $n$, and condition (1) above, along with Stirling's formula, implies that $h_{n} \rightarrow 0$ uniformly on compact subsets of $\mathbf{C}$. Thus our claim follows from Theorem 2.4, with $T=$ differentiation on $H(\mathbf{C}), D_{0}=$ all holomorphic polynomials, and $\left\{x_{n}\right\},\left\{y_{n}\right\}$ replaced by $\left\{g_{n}\right\},\left\{h_{n}\right\}$ respectively.

Now fix $f \in \Gamma(\Delta)$. Then from the definition of $\Gamma(\Delta)$ and the fact that $\rho_{n} \rightarrow \infty$, there is an infinite set $E$ of positive integers $n$ for which

(3) $\left|f^{(n)}-g_{n}\right|<1$ on $\partial \Delta$, and $n \rho_{n} \varepsilon-\rho_{n}>1$.

Fix $n$ in $E$. We claim that $f^{(n)}(\Delta) \supset D\left(0, \rho_{n}\right)$. To see this, fix $a \in D\left(0, \rho_{n}\right)$, and note that conditions (3) above guarantee that for every $z \in \partial \Delta$,

$$
\left|\left(f^{(n)}(z)-a\right)-\left(g_{n}(z)-a\right)\right|<1<n \rho_{n} \varepsilon-|a| \leq\left|g_{n}(z)-a\right|,
$$

so by Rouché's Theorem [4, pp. 125-126], $f^{(n)}$ takes the value $a$ in $\Delta$. 
So far we have shown that for each $f \in \Gamma(\Delta) ; f^{(n)}(\Delta) \supset D\left(0, \rho_{n}\right)$ for infinitely many $n$. To finish the proof, note that the intersection of the sets $\Gamma(\Delta)$, as $\Delta$ runs through all open discs in the plane having rational radii, and centers with rational coordinates, is precisely the set of entire functions obeying condition (2). By the work above, and Baire's Theorem, it is a dense $G_{\delta}$ subset of $H(\mathbf{C})$. This completes the proof.

4. Backward shifts. In this section, $\beta=\{\beta(k): k \geq 0\}$ is a decreasing sequence of positive numbers for which

$$
\sigma=\sup \{\beta(k) / \beta(k+1): k \geq 0\}<\infty .
$$

Following [16] we denote by $H^{2}(\beta)$ the space of power series $f(z)=\sum \hat{f}(n) z^{n}$ for which

$$
\|f\|_{\beta}^{2}=\sum_{k=0}^{\infty}|\hat{f}(k)|^{2} \beta(k)<\infty .
$$

Thus $H^{2}(\beta)$ is a Hilbert space whose elements can be regarded as functions holomorphic on the unit disc $U$. We are going to study the backward shift operator $B$ defined on $H^{2}(\beta)$ by

$$
B f(z)=\sum_{k=0}^{\infty} \hat{f}(k+1) z^{k} \quad\left(f \in H^{2}(\beta)\right) .
$$

An easy computation shows that, thanks to condition (1) above, $B$ is a bounded linear operator on $H^{2}(\beta)$, with norm equal to $\sigma$.

4.1. THEOREM. $H^{2}(\beta)$ has a $B$-universal vector if and only if $\beta(n) \rightarrow 0$ as $n \rightarrow \infty$.

ProOF. Suppose the sequence $\beta$ does not tend to zero. Then, since it is monotonically decreasing, $\delta=\inf \beta(k)>0$. It is a routine exercise to show that for each nonnegative integer $n$,

$$
\left\|B^{n}\right\|=\sup _{k} \beta(k) / \beta(k+n)<\beta(0) / \delta,
$$

so the orbit of each vector in $H^{2}(\beta)$ is bounded, hence no vector can be $B$-universal.

Conversely, suppose $\beta(k) \rightarrow 0$. The forward shift $u$ defined by

$$
u f(z)=\sum_{k=0}^{\infty} \hat{f}(k) z^{k+1} \quad\left(f \in H^{2}(\beta)\right)
$$

is a bounded linear operator on $H^{2}(\beta)$, and $\left\|u^{n} f\right\|_{\beta} \rightarrow 0$ for every $f \in H^{2}(\beta)$ because $\beta(k) \rightarrow 0$. Thus the hypotheses of Theorem 2.2 are satisfied with $X=$ $H^{2}(\beta), D=$ all holomorphic polynomials, $T=B$, and $S=u$. This completes the proof.

4.2. EXAMPLES: BERgman ShIFTS. Suppose $\mu$ is a finite, nonnegative Borel measure on the half-open interval $[0,1)$ which places positive mass on each interval $[a, 1)$ for $0<a<1$. Let $A^{2}(\mu)$ denote the Bergman space of functions $f$ holomorphic in $U$ for which

$$
\|f\|_{\mu}^{2}=(1 / 2 \pi) \int_{0}^{1} \int_{0}^{2 \pi}\left|f\left(r e^{i \theta}\right)\right|^{2} d \theta d \mu(r)<\infty .
$$


Then $A^{2}(\mu)$ coincides (isometrically) with $H^{2}(\beta)$ where $\beta$ is the moment sequence

$$
\beta(k)=\int_{0}^{1} r^{2 k} d \mu(r) \quad(k=0,1,2, \ldots) .
$$

Because $\mu$ places no mass at the point 1 , the sequence $\beta$ decreases monotonically to zero.

\subsection{Proposition. The backward shift $B$ acts boundedly on $A^{2}(\mu)$.}

PROOF. We must show that the moment sequence $\beta$ satisfies condition (1) stated at the beginning of this section. Fix $0<a<1$. Then

$$
\beta(k+1) \geq \int_{a}^{1} r^{2 k+2} d \mu(r) \geq a^{2 k+2} \mu\{[a, 1)\},
$$

and

$$
\begin{aligned}
\beta(k) & <\int_{0}^{a} r^{2 k} d \mu(r)+a^{-2} \int_{a}^{1} r^{2 k+2} d m(r) \\
& \leq a^{2 k} \mu\{[0, a)\}+a^{-2} \beta(k+1) \leq a^{-2}[\mu\{[0, a)\} / \mu\{[a, 1)\}+1] \beta(k+1),
\end{aligned}
$$

so condition (1) is satisfied with $\sigma=a^{-2}[\mu\{[0, a)\} / \mu\{[a, 1)\}+1]$, which is finite because $\mu\{[a, 1)\}>0$. This completes the proof.

4.4 COROLlaRY. For every measure $\mu$ as above, $A^{2}(\mu)$ has $B$ - universal vectors.

4.5. REMARKS. (a) Backward shift on $H^{2}$. If $\beta \equiv 1$, then $H^{2}(\beta)$ is the ordinary Hardy space $H^{2}$ of the unit disc. As pointed out in [5, Theorem 4.1.1], G. Ts. Tumarkin showed that the set of $B$-cyclic vectors is a dense $G_{\delta}$ subset of $H^{2}$. Clearly $B$ has no universal vector in $H^{2}$ (Theorem 4.1), however: for every scalar $a$ of modulus $>1, H^{2}$ has aB-universal vectors. To see this, just apply Theorem 2.2 as in the proof of Theorem 4.1, but with $T=a B$ and $S=a^{-1} u$. Rolewicz [13] has obtained this result by direct construction for the Banach spaces $c_{0}$ and $l^{p}(1 \leq p<\infty)$. Our methods apply in that setting as well.

(b) Weighted shifts. As described in [16] there is a unitary equivalence between the backward shift $B$ on the "weighted spaces" $H^{2}(\beta)$, and weighted backward shifts on the "unweighted" space $H^{2}$. In this regard, our Theorem 4.1 complements a result of Hilden and Wallen [7], who showed that every weighted backward shift has a "supercyclic" vector. A supercyclic vector $x$ for an operator $T$ is one for which the set $\left\{a T^{n} x: a \in \mathbf{C}, n \geq 0\right\}$ is dense in the whole space.

(c) Two-sided shifts. Suppose $\beta=\{\beta(k): k \in \mathbf{Z}\}$ is a two-sided sequence for which $\sup _{k} \beta(k+1) / \beta(k)<\infty$ and $\sup _{k} \beta(k) / \beta(k+1)<\infty$. Then on $L^{2}(\beta)$, the corresponding space of formal Laurent series, the naturally defined bilateral forward and backward shifts, $u$ and $B$ respectively $[16, \S 3]$, are bounded linear operators inverse to each other. Theorem 2.2 applies directly to this situation, and shows that: if $\beta(k) \rightarrow 0$ as $k \rightarrow \infty$ (resp. $k \rightarrow-\infty$ ), then $B$ (resp. $u$ ) has a universal vector.

ADDED IN PROOF. After this paper was accepted for publication, we discovered that Theorem 2.2 had been proved earlier by Carol Kitai as Theorem 1.4 of her unpublished doctoral dissertation Invariant closed sets for linear operators (University of Toronto, 1982). Kitai's dissertation contains many interesting results about 
universal vectors (which she terms orbital vectors). For example, she shows that certain bilateral weighted shifts have universal vectors, yet are not surjective (Theorem 1.10); hence they do not have the right inverse demanded by the hypotheses of our Theorem 2.2. This leads to the study of necessary conditions for an operator to have universal vectors (Chapters 2 and 4). She also studies supercyclic vectors (Chapter 3); and observes that it is possible for an operator to have supercyclic vectors, even though no scalar multiple of the operator has a universal vector.

\section{REFERENCES}

1. G. D. Birkhoff, Démonstration d'un théorème elementaire sur les fonctions entieres, C. R. Acad. Sci. Paris 189 (1929), 473-475.

2. C. Blair and L. A. Rubel, A universal entire function, Amer. Math. Monthly 90 (1983), 331-332.

3. __ A triply universal entire function, Enseign. Math. 30 (1984), 269-274.

4. John B. Conway, Functions of one complex variable, second ed., Springer-Verlaag, New York, 1978 .

5. R. G. Douglas, H. S. Shapiro, and A. L. Shields, Cyclic vectors and invariant subspaces for the backwand shift operator, Ann. Inst. Fourier (Grenoble) 20 (1970), 37-76.

6. A. Edrei and G. R. MacLane, On the zeroes of the derivatives of an entire function, Proc. Amer. Math. Soc. 8 (1957), 702-706.

7. H. M. Hilden and L. J. Wallen, Some cyclic and non-cyclic vectors of certain operators, Indiana Univ. Math. J. 23 (1974), 557-565.

8. W. Luh, On universal functions, Fourier Analysis and Approximation Theory (Proc. Colloq. Budapest, 1976), Vol. II, Colloq. Math. Soc. János Bolyai, no. 19, North-Holland, Amsterdam, 1978, pp. 503-511.

9. __ Universalfunktionen in einfach zusammenhängenden Gebieten, Aequationes Math. 19 (1979), 183-193.

10. G. R. MacLane, Sequences of derivatives and normal families, J. Analyse Math. 2 (1952), $72-87$.

11. G. Pólya, Über die Nulstellen sukzessiver Derivierten, Math. Z. 16 (1922), 36-60.

12. 49 (1943), 178-191.

13. S. Rolewicz, On orbits of elements, Studia Math. 33 (1969), 17-22.

14. Walter Rudin, Functional analysis, McGraw-Hill, New York, 1973.

15. W. P. Seidel and J. L. Walsh, On approximation by Euclidean and non-Euclidean translates of an analytic function, Bull. Amer. Math. Soc. 47 (1941), 916-920.

16. A. L. Shields, Weighted shift operators and analytic function theory, Topics in Operator Theory (C. Pearcy ed.), Amer. Math. Soc., Providence, R. I., 1974.

17. S. M. Duios-Ruis, On the existence of universal functions, Soviet Math. Dokl. 27 (1983), 9-13.

18. Universal functions of the structure of the space of entire functions, Soviet Math. Dokl. 30 (1984), 713-716.

Department of Mathematics, Northern Illinois University, DeKalb, IlliNOIS 60115

Department of Mathematics, Michigan State University, East Lansing, MiCHIGAN 48824 